\title{
Arquivos de Neuro-Psiquiatria
}

Volume 8

Setembro - 1950

Número 3

\section{CHIRURGIE DU SYMPATHIQUE ET TROUBLES CÉRÉBRAUX D'ORIGINE ISCHÉMIQUE}

\author{
P. Wertheimer * \\ L. MANSUY
}

Si les travaux expérimentaux ne permettent pas d'attribuer au sympathique une action certaine sur la circulation dans les petits vaisseaux cérébraux, les constatations cliniques, elles, en maintes occasions, plaident en faveur de l'intervention d'un mécanisme vasomoteur. N'est-ce pas le spasme qui est responsable chez les athéromateux ou les hypertendus de ces hémiplégies ou de ces aphasies dont l'installation brutale évoque l'ictus mais dont la régression rapide est complète sans le moindre déficit permanent? Simple hypothèse? Peut-être, mais qui se trouve confirmée par l'examen ophtalmoscopique lorsque l'artère centrale de la rétine se trouve elle aussi intéressée. Dans un autre ordre d'idées, les neurochirurgiens ont décrit des modifications corticales ou sous-corticales manifestement liées à un dérèglement des fonctions vasomotrices: l'anémie du cortex au cours des crises épileptiques a été souvent relatée et les poussées hypertensives brutales survenant au cours de l'acte chirurgical et déterminant une augmentation rapide du volume de l'encéphale, une turgescence, voire même une rupture des petits vaisseaux se réclament d'une origine sinon exclusivement, du moins très fortement vasomotrice.

L'artériographie cérébrale apporte elle aussi son tribut à ces constatations. Le spasme des troncs artériels ou de leurs branches principales y est nettement visible; il peut même, de par son intensité, simuler, pour l'observateur non averti, l'oblitération complète. Seules des épreuves répétées à quelques jours d'intervalle permettent de lever les doutes dans les cas d'interprétation difficile.

Il est donc permis de se demander puisque la circulation cérébrale possède elle aussi un système vasomoteur, si celui-ci n'est pas susceptible d'influencer la sémeiologie des lésions vasculaires ischémiques de l'encéphale et si, inversement, une thérapeutique sympathique ne serai pas capable, là comme aux membres, d'améliorer des troubles considérés a priori comme liés à un ramollissement cérébral et par conséquent définitifs. Ce sont quelques unes de ces tentatives que nous voudrions évoquer maintenant.

Elles sont déjà anciennes puisqu'une des premières interventions (sinon la première) fut faite par Leriche en 1925: il s'agissait d'une ramisection

* Professeur à la Faculté. Chirurgien des Hôpitaux (Lyon, France). 
cervicale pour hémiplégie récente, le malade fut amélioré mais perdu de vue au bout de deux mois. En 1936, Leriche et Fontaine publièrent l'observation d'une malade, âgée de 58 ans, qui sept jours après une hystérectomie pour fibrome hémorragique, présenta une hémiplégie droite avec aphasie, coma et stertor. Une infiltration stellaire régularisa la respiration et fit dissiper le coma. Trois autres infiltrations, les jours suivants, entrainèrent la rétrocession pratiquement complète de l'hémiplégie et de l'aphasie.

Depuis cette date, des faits de même nature ont été publiés par divers chirurgiens. Dans un article récent Leriche ${ }^{1}$ nous apporte son bilan des interventions sympathiques dans les thromboses récentes et les embolies des vaisseaux cérébraux: il insiste sur la nécessité d'agir rapidement et de façon intensive, le plus souvent par infiltrations stellaires répétées toutes les six heures dans les deux premiers jours, la plupart de ces malades ne pouvant être opérés sans danger. Une de ses observations (publiée en 1936 avec Apfeel) est particulièrement troublante: un malade atteint depuis 18 mois d'angine de poitrine présentait, en outre, un syndrôme de la fossette latérale du bulbe (syndrome de Wallenberg), se traduisant, du côté opposé à la lésion, par une hémiparésie, une hémianesthésie avec dissociation syringomyélique et des douleurs, et, du côté de la lésion, de l'ataxie, de la latero-pulsion et une paralysie des muscles du pharynx, du voile et du larynx. Le malade étant amélioré par les infiltrations stellaires, Leriche réséque le ganglion cervical moyen et l'étoilé: il obtint, outre la disparition des crises angineuses, une regression de l'ataxie et de la déficience cérébelleuse; les troubles sensitifs objectifs s'atténuèrent considérablement, seules persistèrent une hypoesthésie à la douleur dans la moitié droite du corps et une diminution de la sensibilité thermique de la main et de la face postérieure du bras; la déficience pyramidale et l'hypotonie musculaire demeurèrent par contre inchangées. En somme, des troubles déficitaires liés à une thrombose artérielle cérébrale et installés depuis plusieurs mois (ce qui permettait, semble-t'il, de les considérer comme définitifs) régressèrent sous l'influence d'une intervention sympathique.

Sousa Pereira publia au Congrès International de Chirurgie (Londres, 1947) des faits analogues. Sa statistique fait état de quinze observations: trois concernaient des embolies carotidiennes avec hémiplégie et aphasie, traitées l'une 33 heures après l'accident, les autres 2 et 7 ans après, douze des thromboses cérébrales datant de 4 jours à 10 ans. Tous ces malades eurent d'abord des infiltrations stellaires d'épreuve, quatre d'entre eux ne purent être operés en raison de la gravité de leur état mais retirèrent des infiltrations répétées un profit certain, surtout en ce qui concerne le déficit moteur, l'aphasie n'étant que très légèrement améliorée.

Les onze malades restants furent opérés: sympathectomie cervicale inférieure (résection des ganglions moyen, intermédiaire, étoilé et section du nerf vertébral) exécutée chez les cinq derniers de façon bilatérale et complétés pour quatre d'entre eux par une sympathectomie périartérielle bilatérale portant sur la carotide interne et la vertébrale. Les resultats ont 
été variables: 7 malades avaient une hémiplégie droit avec aphasie, 6 virent leur aphasie s'atténuer considérablement tandis que 5 seulement eurent une amélioration de leur déficit moteur; 4 avaient une hémiplégie gauche, un fut un échec presque complet (l'accident vasculaire remontait il est vrai à 10 ans), trois furent améliorés surtout pour le membre inférieur. Il ne semble pas que les sympathectomies bilatérales larges aient donné de meil. leurs résultats.

Reprenant la question sous l'angle expérimental, Sousa Pereira a montré sur des artériographies que la résection de la chaine sympathique cervicale déterminait chez le chien auquel on avait lié les deux carotides in. ternes un développement de la circulation collatérale du côté correspondant. Chez deux malades porteurs de thrombose carotidienne, l'artériographie permit de constater après sympathectomie le même développement de la circula. tion collatérale. Ces constatations radiogıaphiques confirment ce que l'examen ophtalmologique nous avait déjà montré: l'infiltration stellaire faite par exemple pour une rétine pigmentaire ou une atrophie optique augmente de façon très nette la circulation de la papille et de la rétine.

Dans un autre ordre d'idées, Stricker a publié des observations concernant des troubles déficitaires post-traumatiques. La première a trait à un homme de 46 ans, chez lequel une esquille osseuse avait déchiré la duremère, blessé une veine pie-mérienne et lésé le cortex de la région. pariétale droite. Deux ans après, il conservait une monoplégie brachiale gauche complète avec refroidissement de la main, perte de la sensibilité au tact, impotence absolue. Des infiltrations stellaires faites du côté paralysé (dans le but de réchauffer le membre), puis du côté de la lésion, restèrent sans effet. Les infiltrations du ganglion cervical supérieur faites du côté de la lésion cérébrale permirent la récupération de la sensibilité puis la reprise, d'abord très faible, mais progressivement accentuée, de la motilité volontaire; elles eurent même une conséquence curieuse, le réchauffement du membre paralysé, action croisée qui démontre bien l'origine centrale de ces troubles vasomoteurs des hémiplégiques. Après cinq infiltrations, le malade put reprendre son travail; quatre mois plus tard le résultat se maintenait, seuls quelques légers troubles rappelaient l'état antérieur.

Une seconde observation concerne un malade qui, six jours après un traumatisme de la région pariétale gauche, conservait une aphasie. Celle-ci disparut définitivement le lendemain d'une infiltration du ganglion cervical supérieur gauche.

De ces deux observations, nous pouvons rapprocher celle d'un homme de 28 ans que nous avons suivi personnellement. Il conservait six ans après son traumatisme une hémiplégie droite avec dysarthrie. Une artériographie nous montra l'intégrité du système carotidien. 11 eut une résection du gan. glion cervical supérieur et de la partie haute de la chaine cervicale. Mais son indocilité nous empêcha de le suivre et de vérifier par une nouvelle artériographie le résultat obtenu. Deux ans plus tard nous avons eu des nouvelles par téléphone: il se déclarait enchanté du résultat et semblait s'exprimer avec facilité, malheureusement nous n'avons pu apprécier l'évolution de son hémiplégie. 
Les interventions sympathiques peuvent agir non seulement sur les déficits moteurs ou les troubles vasomoteurs, mais également sur les douleurs. Stricker, par une seule infiltration du ganglion cervical supérieur, chez une malade de 66 ans ayant eu le mois auparavant un ictus et qui conservait une monoplégie brachiale avec des douleurs rebelles depuis 2 mois, vit en quelques minutes les douleurs disparaître et les mouvements redevenir possibles.

A ces faits, il convient d'ajouter les constatations faites par Leriche chez deux blessés de guerre porteurs de lésions traumatiques de la carotide primitive. Chez l'un, opéré un an et demi après sa blessure, la résection de la carotide primitive, thrombosée sur presque tout son segment cervical, fit disparaître des douleurs ressenties dans le cou et l'épaule et améliora dans une proportion très notable une hémiparésie et surtout une dysarthrie importante. Sept ans plus tard le blessé avait pu reprendre un travail de cultivateur et parlait presque normalement.

Chez un autre, blessé depuis huit mois, la carotide n'était pas thrombosée mais englobée dans une gangue scléreuse cicatricielle. La libération de l'artère et la résection de l'adventice accentuèrent les résultats déjà obtenus par des infiltrations stellaires, c'est-à-dire améliorèrent l'hémiplégie et firent disparaître une dysarthrie très accentuée. Ce malade, un prêtre, présentait en óutre des troubles de la mémoire, une lenteur considérable de l'idéation et ne pouvait pratiquement plus lire. Après l'intervention il put reprendre ses études au séminaire; revu huit ans plus tard, il exerçait son ministère sans trop de peine.

Ces faits que nous venons de résumer brièvement ne sauraient constituer une statistique, car ils ont été choisis à titre d'exemple parmi les plus favorables; la thérapeutique sympathique ne saurait prétendre influencer toutes les hémiplégies, ni effacer les dégats consécutifs aux embolies ou aux thromboses cérébrales: les échecs y sont beaucoup plus fréquents que les succès. Il nous apparait cependant que, si incomplets qu'ils soient, ces premiers résultats sont riches d'enseignement et nous laissent entrevoir de multiples possibilités.

Sans méconnaître la valeur thérapeutique de cette chirurgie, valeur qui justifie toute tentative, si fragile que soit l'espoir d'un succès, nous voudrions mettre l'accent sur quelques déductions d'ordre plus général. La réalité de perturbations vasomotrices et la possibilité de les traiter par des interventions sympathiques, ne sauraient être mises en doute; pour l'encéphale, comme pour les membres, la lésion vasculaire initiale, thrombose ou embolie, ne reste pas isolée; elle s'accompagne d'un cortège de réactions vasomotrices qui vont aggraver la scène clinique déficitaire. Le champ d'action de ces modifications secondaires est parfois très étendu, débordant largement le territoire tributaire de l'artère lésée et la disproportion entre la lésion initiale minime et ses conséquences vasomotrices est très nette. Il ne s'agit pas là, certes, d'idées nouvelles puisque maints auteurs dont Stricker les avaient mentionnées dans leurs études sur l'hémorragie cérébrale; mais elles étaient restées dans le cadre de spéculations théoriques, alors 
que les faits chirurgicaux leur apportent une confirmation pratique indéniable.

Leur méconnaissance tient surtout à l'impossibilité dans laquelle se trouve le clinicien d'en faire la discrimination. Leur traduction par un syndrome déficitaire d'installation souvent brutale et qui d'emblée atteint son maximum n'offre ancune place à l'analyse. Si l'évolution partiellement régressive permet parfois de soupçonner l'intrication des deux processus, aucun élément ne nous guide pour prévoir l'importance de la récupération et partant pour préciser le pronostic. C'est un des mérites, et non le moindre, de cette chirurgie du sympathique, que de permettre, comme l'a souligné Leriche, l'analyse de ces phénomènes et de dissocier ainsi de l'élément fixe, conséquence de la lésion anatomique, l'élément labile, en rapport avec l'inhibition des fonctions cérébrales, véritable "arrêt fonctionnel" du cerveau, pour reprendre l'expression de Stricker.

Cette étude met en évidence un fait qui heurte nos conceptions habituelles. Instruits dans l'idée de la fragilité du tronc nerveux vis-à-vis de l'ischémie, nous avons tendance à considérer que tout déficit cérébral d'origine vasculaire relève de la destruction définitive des éléments nobles, fibres et cellules, et qu'il sera de ce fait durable, toute suppléance paraissant aléatoire. La transformation brutale de la scène clinique réalisée par la sympathectomie nous oblige à réviser cette conception. Qu'un trouble cérebral, stabilisé depuis plus d'un an et qui à ce titre ne nous paraissait plus réversible, puisse s'atténuer en quelques jours, voilà qui suffit à nous prouver combien nous connaissons mal le mécanisme de ces perturbations vasomotrices. Nous pouvons imaginer qu'à côté de la nécrose cellulaire conséquence de la carence de la circulation vitale, la carence d'une circulation fonctionnelle laisse intacte l'architecture cellulaire et tissulaire mais inhibe son fonctionnement physiologique.

Il se produit là des phénomènes en tous points comparables à ceux que l'on observe au membre inférieur: à la gangrène par arrêt de la circulation vitale s'oppose la claudication intermittent par insuffisance de la circulation fonctionnelle des muscles.

On ne saurait pousser plus loin cette assimilation qui reste un peu simpliste. Il est probable que l'angle vasomoteur sous lequel nous avons envisagé la sympathectomie n'est qu'un des éléments de son action. L'un de nous, étudiant les blessures des nerfs périphériques ou les paralysies faciales traumatiques, a insisté sur le caractère complexe des relations entre les éléments nerveux, leur vascularisation et le système sympatique. Il est vraisemblable que pour le tissu cérébral il en est de même.

Mais si nous voulons apporter une conclusion pratique à ces quelques réflexions, il nous faut souligner ce fait qu'à côté de la pathologie nerveuse conforme aux enseignements de la doctrine anatomo-clinique, il existe une pathologie fonctionnelle qui nous demeure encore pratiquement inconnue. Le seul but de notre travail était d'évoquer les problèmes que soulève son étude, sans aucune prétention à les résoudre. 


\section{BIBLIOGRAPHIE}

Leriche, R. - Infiltrations stellaires, sympathectomies et artériectomie dans les embolies et thromboses des artères cérébrales et après les blessures carotidiennes. Lyon Chir., 44:257-263, 1949.

Leriche, R. et Apfeel - Essai de traitement chirurgical d'un syndrome latéral du bulbe d'origine vasculaire chez un angineux. Presse Méd., 105:2113-2114, 1936.

Leriche, R. et Fontaine, R. - Infiltrations stellaires dans l'embolie cérébrale, dans les spasmes vasculaires post-opératoires de l'encéphale et chez les hémiplégiques. Rev. de Chir., 74:755-758, 1936.

Pereira, A. de Souza - La chirurgie sympathique dans le traitement des embolies et thromboses cérébrales. An. Congrès Internat. de Chir. (Londres), 1947, p. 375-387.

Stricker - Arrêts fonctionnels d'origine cérébrale. Lyon Chir., 44:264-270, 1949.

Wertheimer, P. - À propos de la communication de Stricker. Lyon Chir., 44:254255, 1949.

Wertheimer, P. - Effets des lésions vasculaires sur les lésions nerveuses périphériques. Lyon Chir., 41:385-388, 1946.

Wertheimer, P., Sautot J. et Durand, L. - Le traitement des paralysies faciales traumatiques par les infiltrations sympathiques. Rev. de Chir., 87:129-134, 1949.

41, Avenue de Saxe - Lyon, France. 